\title{
Accurate Prediction of Absolute Acidity Constants in Water with a Polarizable Force Field: Substituted Phenols, Methanol, and Imidazole
}

\author{
George A. Kaminski \\ Department of Chemistry, Central Michigan University, Mount Pleasant, Michigan, 48859 \\ E-mail: kamin1ga@cmich.edu \\ Supporting Information
}

Tables of all the polarizable force field parameters not previously published (for the published parameters see J. Phys. Chem. A, 108, 621-627, 2004) and all refitted modified OPLS-AA parameter values. This material is available free of charge via the Internet at http://pubs.acs.org.

Table S1. Non-Bonded Parameters for the Modified OPLS-AA $\mathrm{C}_{6} \mathrm{H}_{5} \mathrm{O}^{-}$Model

\begin{tabular}{lccc}
\hline \hline Atom & q, electrons & $\sigma, \AA$ & $\varepsilon, \mathbf{~ k c a l} / \mathbf{m o l}$ \\
\hline $\mathrm{C}^{\mathrm{a}}$ & -0.115 & 3.55 & 0.070 \\
$\mathrm{H}^{\mathrm{a}}$ & 0.115 & 2.42 & 0.030 \\
$\mathrm{C}$, connected to $\mathrm{O}^{-}$ & -0.300 & 3.55 & 0.070 \\
$\mathrm{O}^{-}$ & -0.700 & 3.35 & 0.380 \\
\hline
\end{tabular}

\footnotetext{
${ }^{\mathrm{a}}$ Parameters for these atoms are as in the standard OPLS-AA
} 


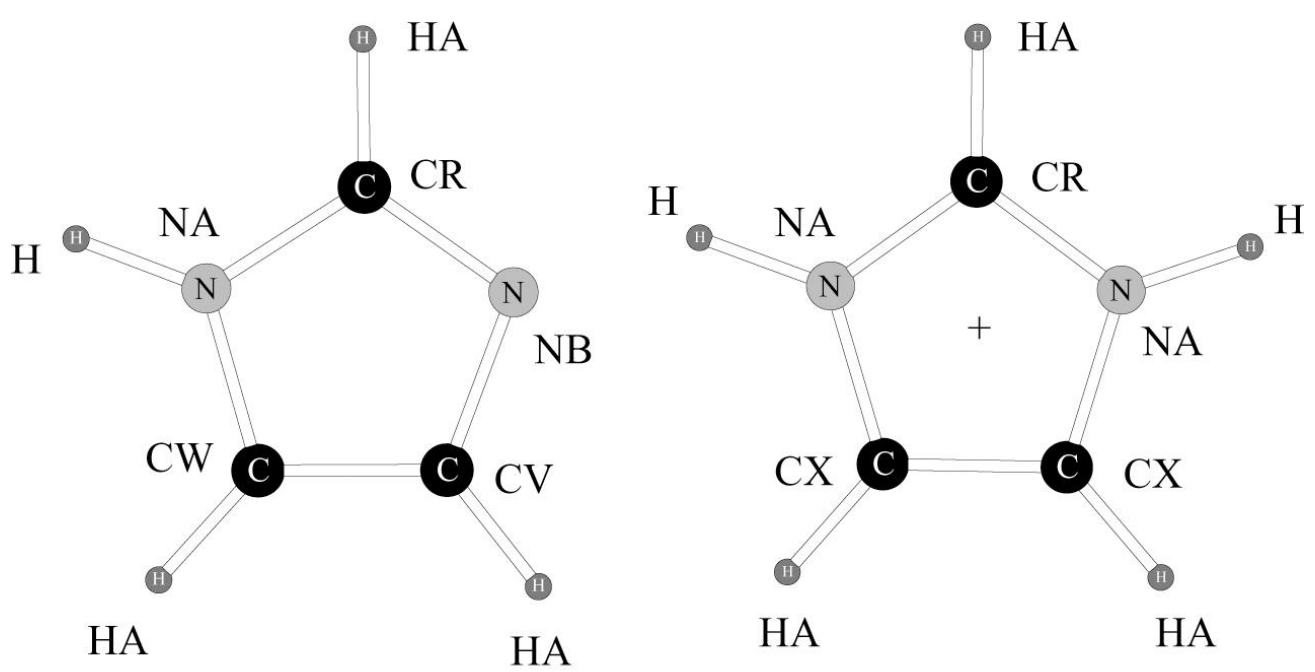

(a)

(b)

Figure S1. Atom type assignments for imidazole (a) and imidazolium (b).

Table S2. Non-Electrostatic PFF Parameters

\begin{tabular}{|c|c|c|c|c|}
\hline Atom & $\underset{\mathrm{kcal} / \mathrm{mol} \cdot \AA^{12}}{\mathbf{1 2}}$ & $\mathrm{B}, \mathrm{kcal} / \mathrm{mol} \cdot \AA^{6}$ & $\mathrm{C}, \mathrm{kcal} / \mathrm{mol}$ & $\alpha, \AA$ \\
\hline $\mathrm{O}$, water & 3500 & 950 & 2470000 & 0.2125 \\
\hline $\mathrm{H}$, water & - & - & - & - \\
\hline $\mathrm{Cl}$ & 24000 & 3550 & 61040 & 0.3415 \\
\hline $\mathrm{N}, \mathrm{NO}_{2^{-}}$ & 4000 & 400 & 18000 & 0.3000 \\
\hline $\mathrm{O}, \mathrm{NO}_{2^{-}}$ & 3500 & 400 & 17000 & 0.2900 \\
\hline $\mathrm{C}$, aromatic & 7500 & 740 & 11600 & 0.3369 \\
\hline $\mathrm{H}$, aromatic & 600 & 20 & 5563.2788 & 0.2385 \\
\hline $\mathrm{O}^{-}, \mathrm{CH}_{3} \mathrm{O}^{-}$ & 3500 & 950 & 16700 & 0.3150 \\
\hline $\mathrm{O}^{-}, \mathrm{C}_{6} \mathrm{H}_{5} \mathrm{O}^{-}$ & 3500 & 950 & 36 & 0.6000 \\
\hline Imidazole CR, CV, CW & 7500 & 740 & 11459.14 & 0.3389 \\
\hline Imidazole NA & 4000 & 900 & 72000 & 0.3030 \\
\hline Imidazole NB & 4000 & 900 & 90000 & 0.2630 \\
\hline $\begin{array}{l}\text { Imidazole/imidazolium } \\
\text { HA }\end{array}$ & 600 & 20 & 5563.2788 & 0.2385 \\
\hline Imidazolium CR, CX & 7500 & 740 & 11459.14 & 0.3389 \\
\hline
\end{tabular}


Tables S3 and S4 list electrostatic parameters. Please note that we used a scaling factor of 1.0 for the 1,4-interactions.

Permanent charge of $-1 \mathrm{e}$ is added to $\mathrm{O}^{-}$atoms. Permanent charges of $+0.5 \mathrm{e}$ are added to the imidazolium NA atoms.

Massless virtual sites representing lone pairs were attached to oxygen atoms at a distance of ca. $0.47 \AA$. For $\mathrm{sp}^{3}$-hybridized oxygens the virtual sites mimic a tetrahedral geometry: they lie in the plane perpendicular to the plane containing the bisector of the angle between the oxygen and its two bond atoms, and make an angle of $\pm \tan ^{-1}\left(2^{1 / 2}\right) \approx \pm 54.74^{\circ}$ from the plane containing these three atoms. For $\mathrm{sp}^{2}-$ hybridized oxygen atoms the virtual sites mimic a trigonal-planar geometry: they lie in the plane containing the oxygen, carbon, and atom bonded to the carbon and make an angle \pm 120 with the $\mathrm{O}$-next atom bond. In addition, virtual sites were placed at the middle of the $\mathrm{C}-\mathrm{H}$ bonds in $\mathrm{CH}_{3}$ - groups. There are no virtual sites attached to any of the $\mathrm{O}^{-}$atoms. All the virtual sites were denoted as $\mathrm{X}$, and had no van-der-Waals parameters or dipoles associated with them. All charge screening lengths for the $X$ type are $0.8 \AA$.

For the fixed bond charge increments - as an example - O_X bci value of 0.1 would mean an electron transfer from $\mathrm{O}$ to $\mathrm{X}$ (negative resulting charge on $\mathrm{X}$ )

Dipole $\chi_{1}$ means internal electrostatic field, resulting in a point "permanent dipole" located on the first atom in the pair, dipole $\chi_{2}$ means internal electrostatic field, resulting in a point "permanent dipole" located on the second atom in the pair. A positive $\chi$ value means field producing a dipole directed toward the other atom in the pair, a negative value - away from the other atom.

Table S3. Atom-Centered PFF Electrostatic Parameters

\begin{tabular}{|c|c|c|c|}
\hline Atom & $\begin{array}{c}\text { inverse } \\
\text { polarizability, } \AA^{-3}\end{array}$ & $\begin{array}{c}\text { charge screening } \\
\text { length, } \AA\end{array}$ & $\begin{array}{c}\text { dipole screening } \\
\text { length, } \AA\end{array}$ \\
\hline $\mathrm{O}$, water & 1.5941955 & 0.4 & 1.8 \\
\hline $\mathrm{H}$, water & 5.9558842 & 0.4 & 1.0 \\
\hline $\mathrm{Cl}$ & 0.70746123 & 0.8 & 2.0 \\
\hline $\mathrm{Cl}$, Phe-Cl & 0.481192 & 0.8 & 2.0 \\
\hline $\mathrm{C}(-\mathrm{Cl})$, Phe-Cl & 0.25814051 & 0.8 & 3.2 \\
\hline $\mathrm{Cl}$, Phe-Cl, deprot. & 0.34810994 & 0.8 & 2.0 \\
\hline $\mathrm{C}(-\mathrm{Cl})$, Phe-Cl, deprot & 0.28449516 & 0.8 & 3.2 \\
\hline
\end{tabular}




\begin{tabular}{|c|c|c|c|}
\hline $\mathrm{N}, \mathrm{NO}_{2}$ & 0.37670703 & 0.8 & 2.0 \\
\hline $\mathrm{O}, \mathrm{NO}_{2}$ & 3.0891268 & 0.4 & 1.8 \\
\hline $\mathrm{N}, \mathrm{NO}_{2}$, Phe- $\mathrm{NO}_{2}$ & 0.42876681 & 0.8 & 2.0 \\
\hline $\mathrm{O}, \mathrm{NO}_{2}$, Phe- $\mathrm{NO}_{2}$ & 2.1495263 & 0.4 & 1.8 \\
\hline $\mathrm{C}\left(-\mathrm{NO}_{2}\right), \mathrm{NO}_{2}, \mathrm{Phe}-\mathrm{NO}_{2}$ & 0.30296187 & 0.8 & 3.2 \\
\hline $\mathrm{N}, \mathrm{NO}_{2}$, Phe- $\mathrm{NO}_{2}$, deprot. & 10.0 & 0.8 & 2.0 \\
\hline $\mathrm{O}, \mathrm{NO}_{2}$, Phe- $\mathrm{NO}_{2}$, deprot. & 10.0 & 0.4 & 1.8 \\
\hline $\begin{array}{l}\mathrm{C}\left(-\mathrm{NO}_{2}\right), \quad \mathrm{NO}_{2}, \quad \mathrm{Phe}-\mathrm{NO}_{2}, \\
\text { deprot. }\end{array}$ & 10.0 & 0.8 & 3.2 \\
\hline $\mathrm{C}$, aromatic & 0.65833113 & 0.8 & 3.2 \\
\hline $\mathrm{H}$, aromatic & - & 0.8 & - \\
\hline $\mathrm{O}^{-}, \mathrm{CH}_{3} \mathrm{O}^{-}$ & 0.71060994 & 0.4 & 2.7 \\
\hline $\mathrm{O}^{-}, \mathrm{C}_{6} \mathrm{H}_{5} \mathrm{O}^{-}$ & 10.0 & 0.4 & 2.7 \\
\hline $\mathrm{C}\left(-\mathrm{O}^{-}\right), \mathrm{C}_{6} \mathrm{H}_{5} \mathrm{O}^{-}$ & 0.49415648 & 0.8 & 3.2 \\
\hline Imidazole CR & 1.0949104 & 0.8 & 2.0 \\
\hline Imidazole CV & 0.89545135 & 0.8 & 2.0 \\
\hline Imidazole CW & 0.89838683 & 0.8 & 2.0 \\
\hline Imidazole NA & 1.065723 & 0.8 & 2.0 \\
\hline Imidazole NB & 0.96836439 & 0.8 & 2.0 \\
\hline Imidazole $\mathrm{H}$ & - & 0.8 & - \\
\hline Imidazole HA (CR) & - & 0.8 & - \\
\hline Imidazole HA (CV) & - & 0.8 & - \\
\hline Imidazole HA (CW) & - & 0.8 & - \\
\hline Imidazolium CR & 1.2811566 & 0.8 & 2.0 \\
\hline Imidazolium CX & 1.1037388 & 0.8 & 2.0 \\
\hline Imidazolium NA & 0.79640759 & 0.8 & 2.5 \\
\hline Imidazolium $\mathrm{H}$ & - & 0.8 & - \\
\hline Imidazolium HA (CR) & - & 0.8 & - \\
\hline Imidazolium HA (CX) & - & 0.8 & - \\
\hline
\end{tabular}


Table S4. Bond-Centered PFF Electrostatic Parameters

\begin{tabular}{|c|c|c|}
\hline Atom & $\begin{array}{c}\text { bond charge increment, } \\
\text { electrons }\end{array}$ & dipole $\chi_{1} / \chi_{2},(\mathrm{kcal} / \mathrm{mol})^{1 / 2} \cdot \AA^{-3 / 2}$ \\
\hline O_H, water & -0.3408 & $-4.0058 /-1.2657$ \\
\hline O_X, water & 0.2963 & - \\
\hline $\mathrm{C} \_\mathrm{Cl}, \mathrm{CH}_{3} \mathrm{Cl}$ & 0.4668011 & $2.3173131 /-3.759353$ \\
\hline C_Cl, Phe-Cl & 0.22672724 & $0.056424142 /-1.2433234$ \\
\hline C_C $(-\mathrm{Cl})$, aromatic & -0.028641086 & $-2.1641778 /-0.057390247$ \\
\hline C_Cl, Phe-Cl, deprot. & 0.15970056 & $-1.0182292 /-1.311033$ \\
\hline C_C(-Cl), aromatic, deprot. & 0.11834804 & $2.2491586 / 1.0265911$ \\
\hline $\mathrm{N} \_\mathrm{O}, \mathrm{CH}_{3} \mathrm{NO}_{2}$ & 0.21121436 & $-0.64251314 / 1.9567756$ \\
\hline $\mathrm{O} \_\mathrm{X}, \mathrm{CH}_{3} \mathrm{NO}_{2}$ & 0.17165281 & - \\
\hline $\mathrm{C} \_\mathrm{N}, \mathrm{CH}_{3} \mathrm{NO}_{2}$ & 0.026501782 & $-0.92316313 / 0.68795406$ \\
\hline N_O, Phe- $\mathrm{NO}_{2}$ & 0.12085609 & $-0.96758285 / 2.0945588$ \\
\hline $\mathrm{O} \_\mathrm{X}, \mathrm{Phe}-\mathrm{NO}_{2}$ & 0.19627169 & - \\
\hline C_N, Phe- $\mathrm{NO}_{2}$ & -0.055202544 & $-0.82532839 / 1.0377354$ \\
\hline C_C $\left(-\mathrm{NO}_{2}\right)$, aromatic & 0.0017087267 & $-1.9154065 / 0.80555694$ \\
\hline N_O, Phe- $\mathrm{NO}_{2}$, deprot. & -0.72997815 & 15.650143 / 33.599459 \\
\hline $\mathrm{O} \_\mathrm{X}$, Phe- $\mathrm{NO}_{2}$, deprot. & 1.0439274 & - \\
\hline C_N, Phe- $\mathrm{NO}_{2}$, deprot. & 1.3345821 & $-16.619679 /-15.960624$ \\
\hline C_C $\left(-\mathrm{NO}_{2}\right)$, Phe-NO ${ }_{2}$, deprot. & -0.42211381 & $-3.8988312 / 16.850815$ \\
\hline $\mathrm{C}_{-} \mathrm{O}^{-}, \mathrm{CH}_{3} \mathrm{O}^{-}$ & -0.094291484 & $4.1601558 /-0.49796643$ \\
\hline $\mathrm{C}_{-} \mathrm{O}^{-}, \mathrm{C}_{6} \mathrm{H}_{5} \mathrm{O}^{-}$ & -0.26046865 & $7.453518 /-0.38055417$ \\
\hline C_C $\left(-\mathrm{O}^{-}\right), \mathrm{C}_{6} \mathrm{H}_{5} \mathrm{O}^{-}$ & 0.0060623769 & $-0.0044337991 /-0.3141662$ \\
\hline Imidazole CV_CW & -0.099080541 & $-0.714825 / 0.9654197$ \\
\hline Imidazole CV_NB & 0.039914687 & $1.4625056 / 4.3887529$ \\
\hline Imidazole CW_NA & -0.13661573 & $-0.52999501 / 1.2571783$ \\
\hline
\end{tabular}




\begin{tabular}{lcc}
\hline Imidazole NB_CR & -0.23462292 & $4.0200861 / 2.4358386$ \\
Imidazole NA_CR & 0.04098804 & $0.25132924 / 0.42476072$ \\
Imidazole NA_H & -0.37082874 & $-1.271898 /-$ \\
Imidazole CR_HA & -0.2374739 & $-2.5878969 /-$ \\
Imidazole CV_HA & -0.18249138 & $-0.58316146 /-$ \\
Imidazole CW_HA & -0.20909493 & $-0.44319061 /-$ \\
Imidazolium CX_CX & 0.0 & $-0.79423178 /-0.79423178$ \\
Imidazolium NA_CR & 0.047455158 & $-1.503238 / 0.0046689745$ \\
Imidazolium NA_CX & 0.017341249 & $-0.39659769 / 0.11709381$ \\
Imidazolium NA_H & -0.11328128 & $1.6481691 /-$ \\
Imidazolium CR_HA & 0.003584685 & $-0.039166135 /-$ \\
Imidazolium CX_HA & -0.055265797 & $0.60737582 /-$ \\
\hline \hline
\end{tabular}

